\title{
Topological magnetic irreversibility in superconducting Pb samples of various shapes
}

\author{
Saül Vélez, ${ }^{*}$ Carles Panadès-Guinart, Guillem Abril, Antoni García-Santiago, ${ }^{\dagger}$ Joan Manel Hernandez, ${ }^{\dagger}$ and Javier Tejada $^{\dagger}$ \\ Grup de Magnetisme, Departament de Física Fonamental, Facultat de Física, Universitat de Barcelona, C. Martí i Franquès 1,
}

Planta 4, edifici nou, 08028 Barcelona, Spain

(Received 16 May 2008; revised manuscript received 25 July 2008; published 1 October 2008)

\begin{abstract}
The magnetic-field dependence of the magnetization of cylinders, disks, and spheres of pure type-I superconducting lead was investigated by means of isothermal measurements of first magnetization curves and hysteresis cycles. Depending on the geometry of the sample and the direction and intensity of the applied magnetic field, the intermediate state exhibits different irreversible features that become particularly highlighted in minor hysteresis cycles. The irreversibility is noticeably observed in cylinders and disks only when the magnetic field is parallel to the axis of revolution and is very subtle in spheres. When the magnetic field decreases from the normal state, the irreversibility appears at a temperature-dependent value whose distance to the thermodynamic critical field depends on the sample geometry. The irreversible features in the disks are altered when they are submitted to an annealing process. These results agree well with very recent highresolution magneto-optical experiments in similar materials that were interpreted in terms of transitions between different topological structures for the flux configuration in the intermediate state. A discussion of the relative role of geometrical barriers for flux entry and exit and pinning effects as responsible for the magnetic irreversibility is given.
\end{abstract}

DOI: 10.1103/PhysRevB.78.134501

PACS number(s): 74.25.Ha, 74.25.Op, 89.75.Kd

One of the most important questions to be yet answered univocally in type-I superconductors is the emergence of irreversibility when the flux enters and leaves the material in the intermediate state. This problem was first studied by Landau ${ }^{1}$ and since then different authors have tried to deepen the understanding of magnetic-flux dynamics and equilibrium structures following both theoretical and experimental approaches. ${ }^{2-10}$ Magnetic hysteresis in type-I superconductors has been attributed historically to impurities, defects, dislocations, and edge barriers. ${ }^{2}$ In the last years, thanks to the emergence of high-resolution magneto-optical imaging, this field has been deeply revised, ${ }^{11-17}$ and different ideas have come to light. This is the case, for example, of the very recent works of Menghini and Wijngaarden ${ }^{14}$ and Prozorov et $a{ }^{15-17}$ In both cases, the main conclusions refer to the existence of topological irreversibility associated to the formation of tubular structures, when the flux enters the material, and laminar patterns in the case of flux exit. In real-time videos shown in Ref. 16, it is also possible to follow the dynamics of the flux structures that help elucidate the physics of irreversibility in the intermediate state of type-I superconductors. In this paper we have extended these studies to investigate the emergence of magnetic irreversibility for flux entering and leaving bulk samples of lead for different configurations of the external magnetic field.

We present here results for a cylinder with a radius of 1.5 $\mathrm{mm}$ and a height of $3 \mathrm{~mm}$ (sample $A$ ), two disks of octagonal cross section with a surface area of $40 \mathrm{~mm}^{2}$ and a thickness of $0.2 \mathrm{~mm}$ (samples $B$ and $C$ ), and a sphere with a radius of $1.5 \mathrm{~mm}$ (sample $D$ ): all of them produced from a commercial rod of extremely pure lead (99.999 at.\%). ${ }^{18}$ The cylinder and the sphere were made by slowly eroding the rod with a whetstone, while the disks were made by cold rolling a cylinder similar to sample $A$ to the desired thickness and cutting out the edges to obtain the final octagonal shape. Sample $C$ was further annealed in glycerol at $290{ }^{\circ} \mathrm{C}$ for $1 \mathrm{~h}$ in nitrogen atmosphere. The magnetic measurements were carried out using a Quantum Design magnetic properties measurement system magnetometer by applying magnetic fields up to 800 Oe and varying the temperature between 2 and $10 \mathrm{~K}$. The low-magnetic-field value of the superconducting transition temperature $T_{c}$ was determined to be $7.2 \pm 0.1 \mathrm{~K}$ from the thermal dependence of the magnetization measured at $10 \mathrm{Oe}$ following zero-field-cooling (zfc) and field-cooling (fc) processes. The low-temperature value of the thermodynamic critical field $H_{c}$ was estimated to be $665 \pm 5$ Oe for all samples from the isothermal first magnetization curve $M_{1 \text { st }}(H)$ measured at $3 \mathrm{~K}$.

Figure 1 shows first magnetization curves and hysteresis cycles measured at $3 \mathrm{~K}$ when the magnetic field was applied either parallel [Fig. 1(a)] or perpendicular [Fig. 1(b)] to the axis of revolution ( $z$ direction) of sample $A$ after a zfc process. Both figures show the presence of a minimum in $M_{1 \mathrm{st}}(H)$ at a magnetic field $H_{c}^{\prime}$ that is taken as the transition point between the Meissner and the intermediate states. The results also indicate the emergence of irreversibility when the magnetic field is parallel to the $z$ direction, whereas the whole $M(H)$ curve is totally reversible when the applied field is perpendicular. The onset of such irreversibility when the magnetic field decreases from $H_{c}$ appears at the so-called irreversibility field $H_{c}^{*}$, at the point where the descending branch of the hysteresis cycle $M_{\mathrm{des}}(H)$ deviates from $M_{1 \text { st }}(H)$. The inset of Fig. 1(a) plots the thermal dependence of $H_{c}^{\prime}, H_{c}^{*}$, and $H_{c}$ determined at temperatures between 3 and $6 \mathrm{~K}$ for which similar experimental facts were observed. The $H_{c}^{*}$ values define a transition line in the $H-T$ diagram that divides the intermediate state in two regions corresponding to reversible and irreversible regimes of flux configuration. It is worth remarking that, in spite of this thermal dependence, the ratio $M_{\text {des }}\left(H_{c}^{\prime}\right) / M_{1 \text { st }}\left(H_{c}^{\prime}\right)$ is almost temperature independent, and a similar behavior is observed for the ratio of the remanent magnetization at zero field $M_{\text {rem }}(0)$ to $M_{1 \mathrm{st}}\left(H_{c}^{\prime}\right)$. This would suggest that the formation and redistribution of 


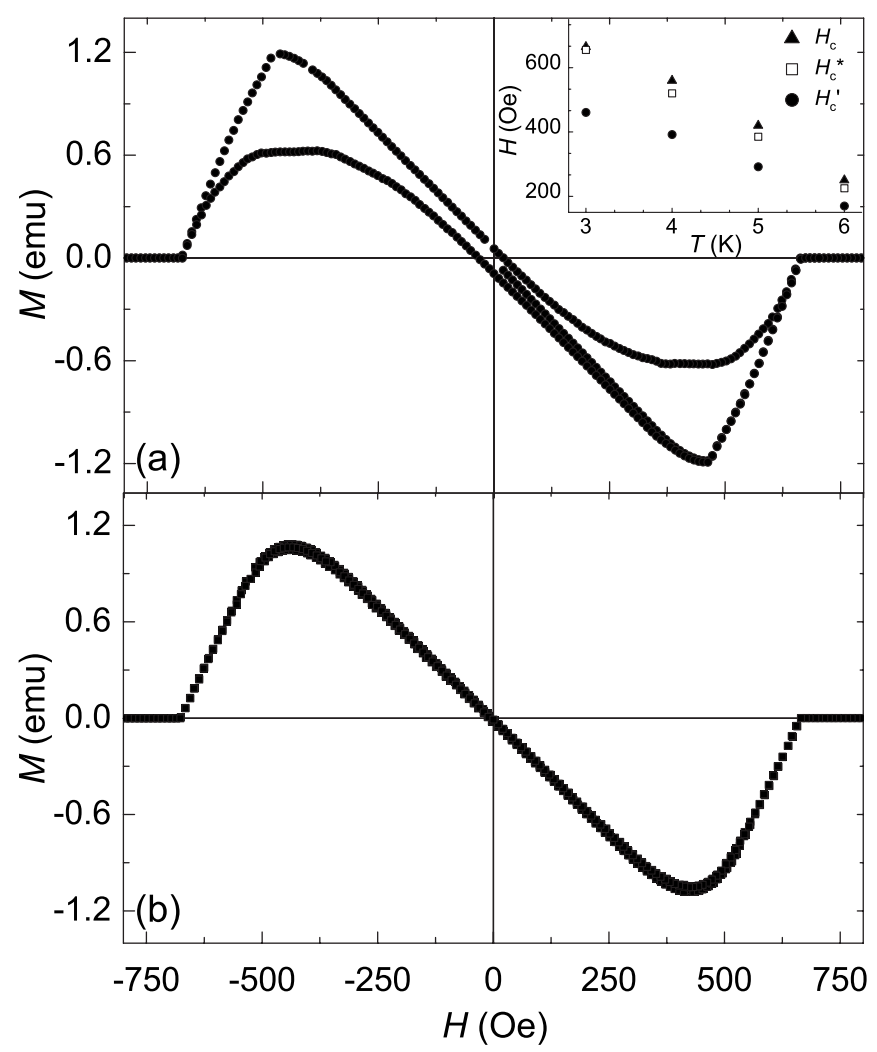

FIG. 1. First magnetization curves and hysteresis cycles $M(H)$ measured at $3 \mathrm{~K}$ on sample $A$ (cylinder) when the magnetic field is applied either parallel (a) or perpendicular (b) to the $z$ direction after a zero-field-cooling process. The inset in the upper panel shows the temperature dependence of the magnetic field at the onset of the intermediate state $\left(H_{c}^{\prime}\right.$, solid circles), the magnetic field at the onset of the irreversibility ( $H_{c}^{*}$, open squares), and the thermodynamic critical field $\left(H_{c}\right.$, solid triangles).

flux structures in the intermediate state of the cylinder should rely on temperature-independent parameters.

Figure 2 shows a minor $M(H)$ loop (open triangles) measured at $3 \mathrm{~K}$ when the magnetic field was applied parallel to the $z$ axis of sample $A$. The full $M(H)$ curve (open circles) is included again to compare both data set. In the minor loop, the magnetic field is first increased from zero to a value close to $H_{c}^{\prime}$ (450 Oe: point 1 in the figure), then is decreased down to -500 Oe (point 2), afterward is increased up to 550 Oe (point 3), and finally is canceled again. The first branch (from zero to point 1) is done in the Meissner regime meaning there is no flux penetration at all and so the second branch of the loop (from point 1 down to point 2) is traced all the way back along the same first branch following a reversible process. The next branch (from point 2 up to point 3) does not follow the same path described by the preceding branch and so irreversibility is established. A similar behavior is observed for the last branch of the loop (from point 3 back to zero field). Each branch is observed to be almost parallel to the one corresponding to the Meissner state until it merges into the full $M(H)$ cycle at a certain point $\left(2^{\prime}\right.$ and $3^{\prime}$ in Fig. 2) from which they become indistinguishable. The fact that each of these branches and the full $M(H)$ curve are the same in a certain region suggests they should share the

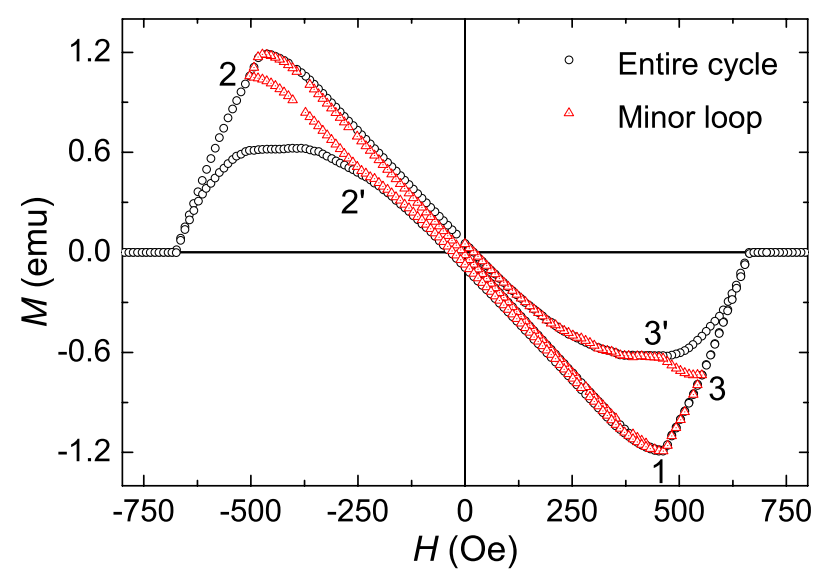

FIG. 2. (Color online) Minor $M(H)$ loop (open triangles) measured on sample $A$ (cylinder) at $3 \mathrm{~K}$ when the magnetic field is applied parallel to the $z$ direction after a zero-field-cooling process. The magnetic field goes from 0 to 450 Oe (point 1), from 450 to -500 Oe (point 2), from -500 to 550 Oe (point 3 ), and back to zero. A full $M(H)$ curve (open circles) is shown superimposed. Points $2^{\prime}$ and $3^{\prime}$ indicate where the minor loop merges into the full curve.

same flux structure in the intermediate state in such region.

Figure 3 presents several minor $M(H)$ loops measured at $3 \mathrm{~K}$ when the magnetic field was applied parallel to the $z$ axis of sample $B$. The entire $M(H)$ cycle (outermost open circles) was first performed after a zfc process. Each minor loop was then obtained going from the normal state $\left(H>H_{c}\right)$ right down to a specific $H_{m}$ value along $M_{\text {des }}(H)$ (first branch) and returning to the initial state by increasing slowly the magnetic field and measuring the magnetization [second branch: $\left.M_{2 \text { nd }}(H)\right]$. The values of $H_{m}$ were equally spaced at $50 \mathrm{Oe}$

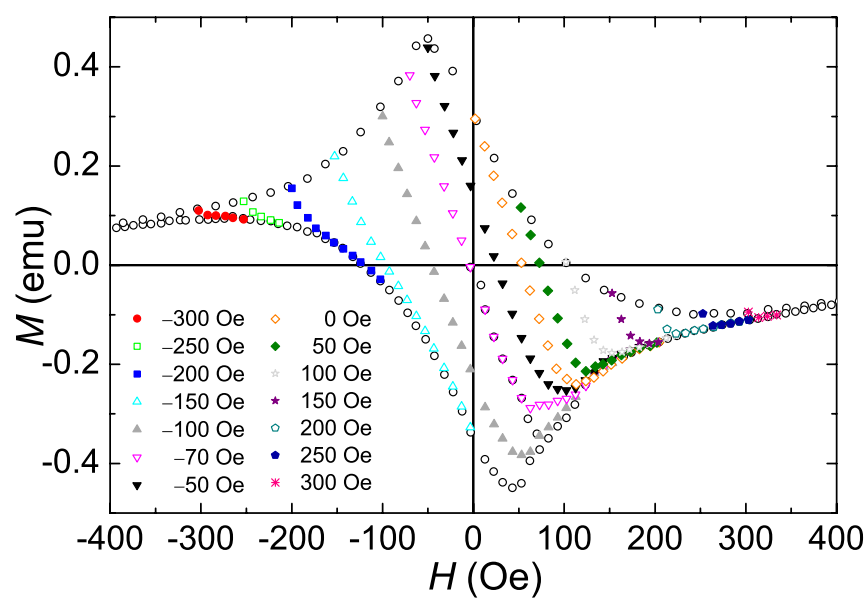

FIG. 3. (Color online) Minor $M(H)$ loops measured on sample $B$ (disk) at $3 \mathrm{~K}$ with the magnetic field applied parallel to the $z$ axis after a zero-field-cooling process. Each loop was obtained starting at the normal state $\left(H>H_{c}\right)$ going right down to a certain $H_{m}$ value and then back to the initial state by slowly measuring the magnetization. The loops are represented with different symbols corresponding to $H_{m}$ values equally spaced 50 Oe in the range from -300 Oe to 300 Oe with an extra value at -70 Oe (see legend for details). The entire $M(H)$ cycle measured at the same temperature is also plotted (outermost open circles). 


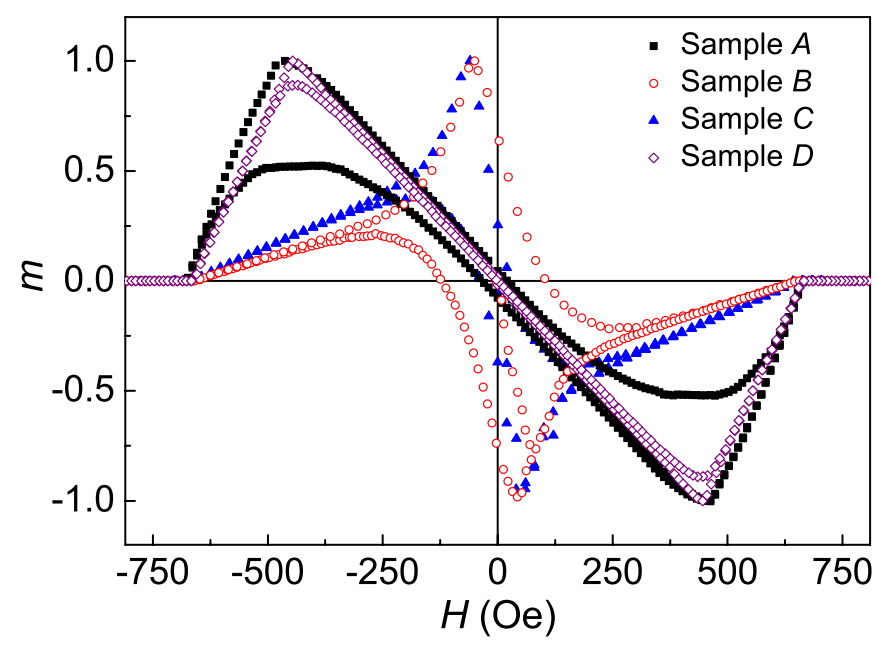

FIG. 4. (Color online) First magnetization curves and hysteresis cycles measured at $3 \mathrm{~K}$ on all samples ( $A$, solid squares; $B$, open circles; $C$, solid triangles; and $D$, open rhombuses) when the magnetic field is applied parallel to the $z$ direction after a zero-fieldcooling process. The reduced magnetization $m$ was defined for each sample as the ratio of the magnetization $M$ to the absolute value at the peak of the ascending branch of the corresponding hysteresis cycle $\left|M_{\text {asc }}\left(H_{p}\right)\right|$.

between -300 and $300 \mathrm{Oe}$, and a minor loop for $H_{m}$ $=-70$ Oe was added to study the zero-field magnetization by returning to the normal state from negative values of the magnetic field. The most important fact is that each $M_{2 \text { nd }}(H)$ merges into the entire $M(H)$ cycle at a certain field value so that a single final magnetic state is defined for all the minor loops. It is also remarkable that each $M_{2 \text { nd }}(H)$ begins parallel to $M_{1 \mathrm{st}}(H)$ and breaks this trend near the merging zone by bending significantly with a shape that depends on the corresponding $H_{m}$ value. Similar results were obtained when sample $C$ was submitted to the same kind of procedures; although in this case, none of the $M_{2 \text { nd }}(H)$ branches did bend prior to merging into the full hysteresis cycle.

First magnetization curves and hysteresis cycles measured on all samples at $3 \mathrm{~K}$ when the magnetic field was applied parallel to the $z$ axis after a zfc process are plotted together in Fig. 4. The difference in the shapes of the curves for samples $A$ and $D$ and the curves for samples $B$ and $C$ reflect how strongly the onset of the intermediate state and the reversible-irreversible transition depends on the sample geometry. In particular, $H_{c}^{\prime}$ was determined to be $450 \pm 5$ Oe for samples $A$ and $D$ and $60 \pm 10$ Oe for samples $B$ and $C$ giving values for the corresponding demagnetizing factors in close agreement with typical figures found for similar samples in this field configuration in the literature $(1 / 3$ for spheres $^{19}$ and cylinders ${ }^{20}$ and $9 / 10$ for disks ${ }^{21}$ ). Results obtained in samples $B$ and $C$ when the external field was applied perpendicular to the $z$ axis (not shown in the figure) proved that the disks did not show magnetic irreversibility in this configuration as it had been also the case for the cylinder [Fig. 1(b)].

For each sample, a reduced magnetization can be defined as the ratio of the magnetization to the absolute value at the peak of the ascending branch of the corresponding hysteresis cycle $m(H) \equiv M(H) /\left|M_{\text {asc }}\left(H_{p}\right)\right|$. The remnant value of this reduced magnetization at zero field $m_{\text {rem }}(0)$ should give an idea of the total amount of trapped flux referred to the corresponding maximum value of flux excluded in the Meissner state. An inspection of Fig. 4 allows to determine $m_{\text {rem }}(0)$ $\simeq 0.70,0.35$, and 0.05 , respectively, for samples $B, C$, and $A$ suggesting that disks have much larger capability than cylinders to trap flux along $M_{\mathrm{des}}(H)$ once the sample has been first driven to the normal state along $M_{1 \mathrm{st}}(H)$. Moreover, a comparison of the values obtained for samples $B$ and $C$ indicates that the main effect of the annealing procedure is to reduce such trapping capability to half its initial value, as well as to modify the shape of the $m(H)$ curve by shifting $H_{c}^{*}$ to lower magnetic fields. As for sample $D$, the $m(H)$ curve has nearly zero remanence $\left[m_{\text {rem }}(0) \simeq 0.01\right]$ and shows only very small irreversibility around $H_{c}^{\prime}\left[m_{\mathrm{des}}\left(H_{c}^{\prime}\right) / m_{1 \mathrm{st}}\left(H_{c}^{\prime}\right)>0.9\right]$ suggesting that spheres are quasiperfectly reversible type-I superconductors - as they are supposed to be-their shape being a particular case of an ellipsoid for which no hysteresis is expected. ${ }^{3}$

The large irreversibility of the intermediate state in samples $B$ and $C$ could be ascribed to the geometrical barriers that are usually present in samples having two parallel surfaces perpendicular to the direction of the magnetic field. ${ }^{22-24}$ This mechanism has been proven experimentally using magneto-optical observations of the flux structure in single-crystalline strips of either lead or indium type-I superconductors having thickness-to-width ratios that ranged roughly from 0.05 to $0.20 .^{25}$ Geometrical barriers, however, should emerge in any nonellipsoidal shape whatever the aspect ratio might be. ${ }^{3-5,15}$ The interplay between such barriers and the applied magnetic field would then also be the reason for the ability of lamellae to leave sample $A$ on decreasing $H$ from the normal state. ${ }^{12}$ In this context, the absence of irreversibility when the applied field is perpendicular to the revolution axis in samples $A$ to $C$ should be a consequence of the fact that the sample edges that generate the geometrical barriers become irrelevant in this configuration. The same should occur (all the more reason) for sample $D$, for which no privileged directions are found, so the geometrical barriers should play a very small role in establishing the equilibrium configuration for the magnetic flux in a sphere.

It might be argued that the irreversibility in our samples could not only be of geometrical nature but it could also be caused by pinning effects. There is strong evidence unfavorable to this interpretation in samples $A$ and $D$ though. First, relaxation experiments performed by starting at any point along the full $M(H)$ cycle and recording the time evolution of the magnetization over $2 \mathrm{~h}$ showed no time dependent phenomena suggesting that every flux pattern along this cycle should be in strong equilibrium in the intermediate state. Second, both samples were produced from the same commercial material following a mechanical procedure that could hardly introduce defects that would act as pinning centers. Third, experimental data at $3 \mathrm{~K}$ taken by measuring the temperature dependence of the $\mathrm{zfc}$ and $\mathrm{fc}$ magnetization at different magnetic fields coincided, respectively, with data in the $M_{1 \mathrm{st}}(H)$ and $M_{\mathrm{des}}(H)$ branches. Finally, in the case of the minor loop of Fig. 2, the curvature and the slope of the third branch (from point 2 to point $2^{\prime}$ ) and fourth branch (from 
point 3 to point $3^{\prime}$ ) are equal to the curvature and slope of the $M_{1 \mathrm{st}}(H)$ curve taken for positive and negative magneticfield intensities up to $\pm H_{c}^{\prime}$ indicating that the changes in the flux trapped at points 2 and 3 are not due to pinning.

Pinning effects, however, should not be completely ruled out in sample $B$ for several reasons. First, relaxation experiments similar to those performed in samples $A$ and $D$ showed relative variations of magnetization of $0.1 \%-1 \%$ over $2 \mathrm{~h}$. Second, the cold rolling process followed to make the sample produced large mechanical stress that could have introduced extended defects that would act as pinning centers in this configuration. Third, although zfc magnetization data measured at different magnetic fields laid on the $M_{1 \mathrm{st}}(H)$ curve at $3 \mathrm{~K}$ and most of the corresponding fc magnetization data coincided with the $M_{\text {des }}(H)$ branch, the low-magneticfield fc points did not match this curve. Finally, although the curvature and the slope of each $M_{2 \text { nd }}(H)$ branch in Fig. 3 are equal to the curvature and slope of the $M_{1 \text { st }}(H)$ curve, they all break this trend near the point at which they merge into the entire hysteresis cycle indicating that the flux configuration could change slightly due to pinning effects. As stated before, the irreversibility in the $M(H)$ curve of sample $C$ is strongly reduced with respect to the same curve for sample $B$ (Fig. 4) suggesting that pinning sources could be partially removed by the annealing process leaving topological effects as the main reason for magnetic hysteresis. It is anyway remarkable that whatever the nature of pinning might be, it was completely absent when the external field was applied perpendicular to the $z$ axis.

In this framework, the magnetic irreversibility in sample $A$ when the applied field was parallel to the revolution axis [Fig. 1(a)] can be understood as follows. At a certain temperature, as the magnetic field increases from $H_{c}^{\prime}$ to $H_{c}$ along $M_{1 \mathrm{st}}(H)$, the magnetic flux enters gradually as bubbles form a tubular pattern. ${ }^{2,15,16}$ This pattern evolves into what has been recently called a suprafroth ${ }^{17}$ that occupies most of the intermediate state in the $H-T$ diagram and finally breaks into a laminar structure close to $H_{c}$ in order to increase the dimensions of the normal regions. When the magnetic field starts to decrease from the normal state for intensities in the reversible region $H_{c}^{*}<H<H_{c}$, the lamellae are able to escape from the cylinder. As the magnetic field drives the sample into the irreversible region, flux remains trapped in a laminar regime that eventually would evolve into a tubular structure close to zero magnetic field in an effort to exit the sample. ${ }^{15}$ When the applied field was perpendicular to the $z$ axis [Fig. 1(b)], the laminar pattern should turn easily into a tubular structure when the magnetic field just decreases from $H_{c}$. This would support the claim that the tubular pattern should be the equilibrium ground state in the intermediate regime and should only depend on temperature and magnetic field, but not on the magnetic history of the sample. ${ }^{12,15}$

For samples $B$ and $C$ in the parallel orientation, the entire $M(H)$ cycle (Fig. 4) may be divided in four regions determined by the two peaks of magnetization at $\pm H_{p}$ with $\left|H_{p}\right|$ $\sim 50$ Oe and at $\pm H_{c}$. Let us suppose we start to decrease the magnetic field from $+H_{c}$. In the first region down to $-H_{p}$, the normal zones would have a laminar structure as reported in the videos of Ref. 16, for instance. The transition from the laminar to the tubular regime that in sample $A$ takes place near zero field would not occur here until a certain negative value for the magnetic field is reached as a consequence of the large amount of flux trapped at zero field. From this transition point down to $-H_{c}$, the system should be in a tubular regime. Analogous features would correspond to the other two regions the sample crosses as the magnetic field increases: From $-H_{c}$ to $+H_{p}$, the sample would be in a laminar regime and from then on the flux pattern should be tubular up to $+H_{c}$. We should not disregard the fact that the structure of the flux pattern in each regime could be somehow blurred by the possible presence of pinning in these samples.

Focusing on the minor loop of sample $A$ (Fig. 2), a transition region from the tubular to the laminar regime should exist somewhere between the point at which the variation of magnetic field changes sign (points 2 or 3 in the figure) and the point at which the subsequent branch of the loop merges into the full $M(H)$ cycle (points $2^{\prime}$ or $3^{\prime}$ in the figure) because at each of these merging points the flux configuration corresponds to the laminar regime. ${ }^{15,16}$ The transitions in the minor loops of sample $B$ (Fig. 3) are not that simple and should be accordingly divided into two kinds as the magnetic field increases: For $H_{m} \leq-150$ Oe, the disk should change from the tubular to the laminar regime; while for $H_{m}$ $\geq 50 \mathrm{Oe}$, the flux pattern should change from laminar to tubular. These transitions should occur at certain magneticfield intensities between the $H_{m}$ value and the point at which the corresponding $M_{2 \text { nd }}(H)$ merges into the full $M(H)$ cycle. A possible explanation could be the dependence of the magnetic contribution of the superconducting zones on the modulus of the magnetic field $|H|$. When the flux is in a tubular state $\left(H_{m} \leq-150 \mathrm{Oe}\right)$ and $|H|$ decreases, the trapped flux should remain constant but the superconducting zones should decrease and so would their contribution to the total magnetization. This would allow the union between different tubular regions into laminar patterns. In the opposite case, when the flux is in a laminar state $\left(H_{m} \geq 50 \mathrm{Oe}\right)$ and $|H|$ increases, the superconducting zones should grow among the laminar structures and so the latter would break into tubular patterns.

The minor loops performed for $H_{m}$ values between -150 and 50 Oe would contain transitions that could not be so well defined in this framework. For instance, $M_{2 \text { nd }}(0)=0$ is found for the minor loop corresponding to $H_{m}=-70 \mathrm{Oe}$, but this branch does not coincide entirely with $M_{1 \text { st }}(H)$ at positive intensities because a bending appears just before $M_{2 \text { nd }}(H)$ merges into the full $M(H)$ cycle. This could be explained if the system would have trapped flux in opposite senses in the initial state so that a complete cancellation would occur at zero field as the magnetic field increases. This would also suggest that the way each $M_{2 \text { nd }}(H)$ merges into the full hysteresis cycle depends strongly on the flux trapped at the $H_{m}$ state.

To summarize, magnetic irreversibility emerging from the difference in topologies upon flux entry and exit in the intermediate state of type-I superconductors has been proven in pure lead samples of different shapes. Our results corroborate that geometrical barriers are mainly responsible for the magnetic hysteresis of disks and cylinders. Their influence is strongly related with the shape of the sample and the direc- 
tion of the magnetic field. The geometrical barriers control the transition between lamellae and tubes and also the capability of the sample to expel the trapped flux in temperatureindependent processes. The results also support the absence of relevant pinning effects in cylinders and spheres and suggest that the tubular structure should be the equilibrium ground state for the flux configuration in these geometries. To elucidate the relevance of pinning effects in disks, alternative procedures for sample fabrication should be sought in order to minimize mechanical stress and other sources of defects.

Further experiments based on magneto-optical techniques should try to focus on minor loops to study whether the transitions between the different structures in cylinders are gradual or abrupt and elucidate the magnetic field at which such transitions occur. In the case of disks, it would be of great interest to determine the precise structure of flux patterns in the different ranges of magnetic field and to study the region where the minor loops leave linearity and join the entire $M(H)$ cycle, particularly for those loops showing vaguely defined transitions.

S.V., C.P.-G., and G.A. acknowledge support from the Spanish Government under Project No. MAT2005-06162. J.M.H. acknowledges support from Ministerio de Educación y Ciencia and Universitat de Barcelona. A.G.-S. acknowledges support from Universitat de Barcelona. This work was financially supported by the aforementioned project. *svelez@ubxlab.com

${ }^{\dagger}$ Also at Institut de Nanociència i Nanotecnologia IN2UB, Universitat de Barcelona, c. Martí i Franquès 1, 08028 Barcelona, Spain.

${ }^{1}$ L. D. Landau, Zh. Eksp. Teor. Fiz. 7, 371 (1937); L. Landau, Nature (London) 141, 688 (1938).

${ }^{2}$ For an overview of works performed up to the late sixties, see J. D. Livingston and W. DeSorbo, in Superconductivity, edited by R. D. Parks (Dekker, New York, 1969), Vol. 2, p. 1235.

${ }^{3}$ J. Provost, E. Paumier, and A. Fortini, J. Phys. F: Met. Phys. 4, 439 (1974).

${ }^{4}$ A. Fortini and E. Paumier, Phys. Rev. B 14, 55 (1976).

${ }^{5}$ A. Fortini, A. Hairie, and E. Paumier, Phys. Rev. B 21, 5065 (1980).

${ }^{6}$ H. Frahm, S. Ullah, and A. T. Dorsey, Phys. Rev. Lett. 66, 3067 (1991).

${ }^{7}$ R. E. Goldstein, D. P. Jackson, and A. T. Dorsey, Phys. Rev. Lett. 76, 3818 (1996).

${ }^{8}$ H. Bokil and O. Narayan, Phys. Rev. B 56, 11195 (1997).

${ }^{9}$ A. T. Dorsey and R. E. Goldstein, Phys. Rev. B 57, 3058 (1998).

${ }^{10}$ A. Cebers, C. Gourdon, V. Jeudy, and T. Okada, Phys. Rev. B 72, 014513 (2005).

${ }^{11}$ M. Menghini and R. J. Wijngaarden, Phys. Rev. B 72, 172503 (2005).

${ }^{12}$ R. Prozorov, R. W. Giannetta, A. A. Polyanskii, and G. K. Per- kins, Phys. Rev. B 72, 212508 (2005).

${ }^{13}$ C. Gourdon, V. Jeudy, and A. Cebers, Phys. Rev. Lett. 96, 087002 (2006).

${ }^{14}$ M. Menghini and R. J. Wijngaarden, Phys. Rev. B 75, 014529 (2007).

${ }^{15}$ R. Prozorov, Phys. Rev. Lett. 98, 257001 (2007).

${ }^{16}$ See EPAPS Document No. E-PRLTAO-98-024726 for real-time videos on flux penetration and exit in lead samples. For more information on EPAPS, see http://www.aip.org/pubservs/ epaps.html.

${ }^{17}$ R. Prozorov, A. F. Fidler, J. R. Holberg, and P. C. Canfield, Nat. Phys. 4, 327 (2008).

${ }^{18}$ Alfa Aesar 42691 (lead rod, $6.35 \mathrm{~mm}$ diameter).

${ }^{19}$ J. A. Osborn, Phys. Rev. 67, 351 (1945).

${ }^{20}$ M. Sato and Y. Ishii, J. Appl. Phys. 66, 983 (1989).

${ }^{21}$ J. Helszajn, IEEE Microw. Wirel. Compon. Lett. 15, 392 (2005).

${ }^{22}$ E. Zeldov, A. I. Larkin, V. B. Geshkenbein, M. Konczykowski, D. Majer, B. Khaykovich, V. M. Vinokur, and H. Shtrikman, Phys. Rev. Lett. 73, 1428 (1994).

${ }^{23}$ M. Benkraouda and J. R. Clem, Phys. Rev. B 53, 5716 (1996).

${ }^{24}$ M. Benkraouda and J. R. Clem, Phys. Rev. B 58, 15103 (1998).

${ }^{25}$ H. Castro, B. Dutoit, A. Jacquier, M. Baharami, and L. Rinderer, Phys. Rev. B 59, 596 (1999). 